\title{
Empirical Paradigm: Position Paper
}

\author{
Carolyn B. Seaman
}

The distinction between "exploratory" and "confirmatory" work is crucial but is too often blurred in current literature. The term "exploratory" refers to empirical work that has as a goal the discovery of new and unforeseen insight. "Confirmatory" research, on the other hand, normally begins with some type of hypothesis or proposition (that has some type of support in previous literature), the confirmation of which is the aim of the study.

My observation in recent literature, however, is that the term "exploratory" is often used as an apologia for a study that is smaller, has less significant results, or is less rigorous than the authors had hoped. This implies that exploratory work has lower standards for size, significance, and rigor. This is not the case. One could argue that the standards for exploratory work should in fact be higher than that for confirmatory. Confirmatory results are rarely interpreted on their own; they are compared to, and interpreted in light of, a body of related work that together sheds light on the validity of a hypothesis. However, exploratory studies are intended to be used to justify further expenditure of resources in the form of future studies. Thus a poorly executed exploratory study potentially has a greater negative impact on the field than a poorly executed confirmatory study. This is not to say that there is no room in our body of literature for reporting exploratory studies that are not perfect. The key is to present our work along with its limitations (which, as an aside, I believe we are getting much better at) and to recognize the contributions of results that we did not expect.

It is my position that qualitative research methods are appropriate for both exploratory and confirmatory research. Further, the most appropriate approach to addressing nearly any research problem in either category is a combination of the two. However, qualitative studies are often confined to the exploratory side of the equation. I would argue that this is due more to the comfort level of reviewers and researchers in our community than on any inherent methodological limitations. There exist guidelines, techniques, and tools from other disciplines for qualitative confirmatory analysis. These would need tailoring and translating to be useful for software engineering and norms would have to emerge about how such studies should be presented. But the largest obstacle to the use of qualitative methods for confirmatory analysis is our own stomach for accepting the idea of hypothesis testing without $\mathrm{p}$-values and tables of statistical summaries. This requires some pioneering examples of rigorous qualitative confirmatory analysis that are both well presented and relevant to our discipline. 